\title{
A comparison of the stages at which cancer is diagnosed in physicians and in the general population in Taiwan
}

\author{
Yueh-Han Hsu MD MPH, Pei-Tseng Kung ScD, Yueh-Hsin Wang MHA, Yao-Mao Chang LLM PhD, \\ Wen-Chen Tsai DrPH
}

Competing interests: None declared.

This article has been peer reviewed.

Accepted: June 10, 2015

Online: July 20, 2015

Correspondence to:

Wen-Chen Tsai:

wtsai@mail.cmu.edu.tw

CMAJ 2015. DOI:10.1503/ cmaj.141432

\begin{abstract}
Background: Previous investigations have reported that physicians tend to neglect their own health care; however, they may also use their professional knowledge and networks to engage in healthier lifestyles or seek prompt health services. We sought to determine whether the stage at which cancer is diagnosed differs between physicians and nonphysicians.
\end{abstract}

Methods: We conducted a nationwide matched cohort study over a period of 14 years in Taiwan. We accessed data from two national databases: the National Health Insurance Research Database and the Taiwan Cancer Registry File. We collected data on all patients with the 6 most common cancers in Taiwan (hepatoma, lung, colorectal, oral, female breast and cervical cancer) from 1999 to 2012. We excluded patients less than 25 years of age, as well as those with a history of organ transplantation, cancer or AIDS. We used propensity score matching for age, sex, residence and income to select members for the control (nonphysicians) and experimental (physicians) groups at a 5:1 ratio. We used $\chi^{2}$ tests to ana- lyze the distribution of incident cancer stages among physicians and nonphysicians. We compared these associations using multinomial logistic regression. We performed sensitivity analyses for subgroups of doctors and cancers.

Results: We identified 274003 patients with cancer, 542 of whom were physicians. After propensity score matching, we assigned 536 physicians to the experimental group and 2680 nonphysicians to the control group. We found no significant differences in cancer stage distributions between physicians and controls. Multinomial logistic regression and sensitivity analyses showed similar cancer stages in most scenarios; however, physicians had 2.64-fold higher risk of having stage IV cancer at diagnosis in cases of female breast and cervical cancer.

Interpretation: In this cohort of physicians in Taiwan, cancer was not diagnosed at earlier stages than in nonphysicians, with the exception of stage IV cancer of the cervix and female breast.
$\mathrm{T}$ he health of physicians is vital to health care systems. Physicians who are unwell mentally or physically are prone to providing suboptimal patient care. ${ }^{1}$ Several studies have investigated the risk of cancer for doctors with inconclusive findings $;{ }^{1-4}$ few investigations have addressed whether cancer is diagnosed at earlier stages in physicians.

Previous investigations have reported that physicians tend to neglect their own physical examinations and, once sick, procrastinate seeking medical treatment. ${ }^{5-8}$ However, doctors may use their own professional knowledge and network to engage in healthy lifestyles or seek prompt health services in ways that reduce their risk of illness. ${ }^{9-11}$

Factors protecting people from advanced cancer stages include attending screening services ${ }^{12-14}$ and access to physicians. ${ }^{15,16}$ Delayed cancer diagnoses lead to poorer outcomes. We sought to compare the incident cancer stages of the 6 most common cancers between physicians and nonphysicians in Taiwan to determine whether physicians' cancers were diagnosed at earlier or later stages than nonphysicians' cancers.

\section{Methods}

\section{Study setting and data sources}

We conducted a nationwide propensity score matched cohort study using data from two national databases in Taiwan.

The National Health Insurance Research Database is maintained by the Taiwan National Health Research Institutes. The national insurance program is a mandatory program providing comprehensive medical care services that began in 1995; by the end of 2014, more than $99.6 \%$ of the people in Taiwan were enrolled. ${ }^{17}$ More than 93\% of Taiwan's hospitals and clinics have contracts with the National Health Insurance system. ${ }^{17}$ 
The database includes registry data of beneficiaries, claims files and the registry of health personnel. The health personnel in the database include Western physicians, practitioners of traditional Chinese medicine, dentists, pharmacists, nurses, medical laboratory technicians and physical and occupational therapists. The claims data are periodically reviewed to ensure accuracy.

The Taiwan Cancer Registry File is authorized by the Taiwan Health Promotion Administration. ${ }^{12,18}$ The registry consists of patient data, principles of diagnosis confirmation, staging, treatment information, recurrence status and hospital information, including principles of diagnosis confirmation and staging. The rules for cancer staging in the database follow the regulations from the American Joint Committee on Cancer. ${ }^{12,19-21}$ Using this database, we identified the incident cancer stage of each patient from stage 0 to stage IV.

Data from the two registries were linked through an encrypted unique identification number to protect personal privacy and to provide patient-level information. We retrieved diagnostic information according to the International Classification of Diseases, 9th revision, Clinical Modification (ICD-9-CM) codes.

This study was approved by the Institutional Review Board of Ditmanson Medical Foundation Chia-Yi Christian Hospital and China Medical University Hospital (IRB no. CYCH-101042, CMUH 103-REC-003).

\section{Study cohort}

The long form of the Taiwan Cancer Registry File contains information on the 6 most common cancers in Taiwan (lung cancer [ICD-9-CM code 162], hepatoma [ICD-9-CM code 155], colorectal cancer [ICD-9-CM codes 153, 154], oral cancer [ICD-9-CM codes 140-149], female breast cancer [ICD-9-CM code 174] and cervical cancer [ICD-9-CM codes 180-182]). ${ }^{20}$ We linked the data in the National Health Insurance Research Database with those in the long form from Jan. 1, 1999 (the index date), to the end of 2012 (the latest available data) and identified all incident patients with 1 of the 6 most common cancers; we further selected those patients who were physicians (including physicians who practise Western medicine [Western physicians], practitioners of traditional Chinese medicine and dentists) as the physician cohort. Patients who were not physicians were assigned to the control group. We excluded patients who were less than 25 years of age (the youngest possible age of a physician in Taiwan), as well as patients with a history of organ transplantation (ICD-9-CM code V42), AIDS (ICD-9-CM codes 042, V08) or any cancer before the index date. For each patient in the physician group, we assigned 5 patients to the control group using the propensity score match with age, sex, residence and income.

\section{Covariates}

We categorized residence into 7 levels, with level 1 the highest degree of urbanization..$^{22}$ To simplify the comparisons, level 1 was chosen as the reference, and the other 6 levels were divided into 3 groups (levels 2 and 3, levels 4 and 5, and levels 6 and 7). We used the modified Charlson comorbidity index ${ }^{23}$ to classify the severity of comorbidity, subgrouping the scores as follows: $0,1-3,4-6$, and $\geq 7$. Higher scores denote greater comorbidity. Presence of catastrophic illness was classified as yes or no. Injuries and illnesses defined as catastrophic in Taiwan include 31 categories of major illnesses (e.g., cancer, end-stage renal disease, hemophilia) for which patients are exempt from copayment and may thus avoid financial hardship. ${ }^{24}$

\section{Statistical analysis}

Our primary outcome was the stage at which cancer was diagnosed (stage 0 to stage IV). We used the $\chi^{2}$ test to analyze the sociodemographic characteristics of all patients before and after matching. We then used the $\chi^{2}$ test to compare the distribution of cancer stages among physicians and nonphysicians with respect to age, sex, income, residence, catastrophic illness and severity of comorbidity.

Because our study outcome was an ordinal outcome, we tested whether the ordinal logistic regression model was suitable. The ordinal logistic regression model assumes that the odds ratios (ORs) estimating the effect of an exposure variable (i.e., physicians or nonphysicians) for any comparison (stage $0 \mathrm{v}$. stage $\geq$ I, stage $<$ II v. stage $\geq$ II, stage $<$ III v. stage $\geq$ III, stage $<$ IV v. stage IV) will be similar regardless of the cut-off point. ${ }^{25}$ Thus, we conducted a score test, to assess whether the model constrained by the ordinal logistic regression model assumption significantly differed from the multinomial logistic regression model in which the OR parameters were not constrained by the proportional odds assumption. The score test results showed significant differences between the 2 models $(p<0.05)$, suggesting that the proportional odds assumption was violated.

We performed the multinomial logistic regression model instead of the ordinal logistic regression model in our analyses. In addition, we conducted sensitivity analyses for the odds in physician subgroups (all physicians v. Western physicians only) and cancer subgroups (all 6 cancers; 4 cancers excluding the 2 female-only 
cancers; the 2 female-only cancers). We then used the Hosmer-Lemeshow test to check for goodness-of-fit of the multinomial logistic regression model. We used sex, age, residence and monthly income as the control variables. The $p$ value of Hosmer-Lemeshow test for all 24 multinomial logistic regression models were not significant, which meant that these models were acceptable. We performed all statistical analyses using SAS 9.3 software (SAS Institute, Cary, NC, USA) for Windows. We set the level of significance at 0.05 , and all tests were 2-tailed.

\section{Results}

We collected data for 274003 patients, 542 of whom were physicians. After propensity score matching, we assigned 536 physicians to the experimental group and 2680 nonphysicians to the control group (Table 1). The distributions of cancer stages among the physicians and the nonphysicians under bivariate analysis were similar in terms of sex, age, residence, income, catastrophic illness and severity of comorbidity (Table 2).

Unadjusted multinomial logistic regression analysis showed no significant differences in most scenarios, with the exception of the 2 female-only cancers. For female breast and cervical cancer, physicians had 2.64-fold higher risk of having stage IV cancer at diagnosis than having stages 0 to III (Table 3). After adjusting for age, sex (as necessary), income and residence (Table 4), the odds of having significant differences in cancer stages remained similar, with the exception of the 2 female-only cancers. Physicians had 2.37- to 2.46-fold higher risks of having higher stages of female breast and cervical cancer at diagnosis, although the results of the sensitivity analysis involving Western physicians were nonsignificant.

\section{Interpretation}

Our findings suggest that physicians are not more likely to have an earlier stage of cancer at diagnosis

Table 1: Characteristics of participants before and after propensity score matching

\begin{tabular}{|c|c|c|c|c|c|c|c|c|}
\hline \multirow[b]{2}{*}{ Variable } & \multicolumn{3}{|c|}{ Before matching, no. (\%) } & \multirow[b]{2}{*}{$p$ value } & \multicolumn{3}{|c|}{ After matching, ${ }^{*}$ no. (\%) } & \multirow[b]{2}{*}{$p$ value } \\
\hline & $\begin{array}{c}\text { Total } \\
(n=274003)\end{array}$ & $\begin{array}{c}\text { Nonphysicians } \\
(n=273 \text { 461) }\end{array}$ & $\begin{array}{l}\text { Physicians } \\
(n=542)\end{array}$ & & $\begin{array}{c}\text { Total } \\
(n=3216)\end{array}$ & $\begin{array}{l}\text { Nonphysicians } \\
(n=2680)\end{array}$ & $\begin{array}{l}\text { Physicians } \\
(n=536)\end{array}$ & \\
\hline Sex & & & & $<0.001$ & & & & 0.97 \\
\hline Male & $133538(48.7)$ & $133138(48.7)$ & $400(73.8)$ & & $2369(73.7)$ & 1975 (73.7) & $394(73.5)$ & \\
\hline Female & 140465 (51.3) & $140323(51.3)$ & $142(26.2)$ & & $847(26.3)$ & $705(26.3)$ & $142(26.5)$ & \\
\hline Age, yr & & & & 0.001 & & & & 0.99 \\
\hline $25-44$ & 40881 (14.9) & 40781 (14.9) & $100(18.5)$ & & $597(18.6)$ & $498(18.6)$ & $99(18.5)$ & \\
\hline $45-54$ & $61617(22.5)$ & $61485(22.5)$ & $132(24.4)$ & & $773(24.0)$ & $642(24.0)$ & $131(24.4)$ & \\
\hline $55-64$ & $60294(22.0)$ & $60159(22.0)$ & $135(24.9)$ & & $791(24.6)$ & $658(24.5)$ & $133(24.8)$ & \\
\hline$\geq 65$ & $111211(40.6)$ & $111036(40.6)$ & $175(32.3)$ & & $1055(32.8)$ & $882(32.9)$ & $173(32.3)$ & \\
\hline Residence, lev & of urbanizatio & & & $<0.001$ & & & & 1.000 \\
\hline 1 & $78010(28.5)$ & $77811(28.5)$ & $199(36.7)$ & & $1199(37.3)$ & $1000(37.3)$ & $199(37.1)$ & \\
\hline 2 and 3 & $122463(44.7)$ & $122231(44.7)$ & $232(42.8)$ & & $1393(43.3)$ & $1161(43.3)$ & $232(43.3)$ & \\
\hline 4 and 5 & $49249(18.0)$ & $49175(18.0)$ & $74(13.6)$ & & $440(13.9)$ & $366(13.7)$ & $74(13.8)$ & \\
\hline 6 and 7 & 24281 & 24244 (8.9) & $37(6.8)$ & & $184 \quad(5.7)$ & $153(5.7)$ & $31(5.8)$ & \\
\hline Monthly incor & e (NT\$) & & & $<0.001$ & & & & 1.000 \\
\hline$\leq 17280$ & 15369 (5.6) & 15343 (5.6) & $26(4.8)$ & & $156 \quad(4.8)$ & $130 \quad(4.8)$ & $26(4.8)$ & \\
\hline $17281-36300$ & 209179 (76.3) & $209074(76.5)$ & $105(19.4)$ & & $630(19.6)$ & $525(19.6)$ & 105 (19.6) & \\
\hline $36301-45800$ & 22396 (8.2) & 22302 (8.2) & $94(17.3)$ & & $568(17.7)$ & $474(17.7)$ & $94(17.5)$ & \\
\hline $45801-57800$ & $10929(4.0)$ & 10889 & $40(7.4)$ & & $239 \quad(7.4)$ & $199(7.4)$ & $40 \quad(7.5)$ & \\
\hline $57801-72800$ & 9362 (3.4) & 9267 (3.4) & 95 (17.5) & & 575 (17.9) & 480 (17.9) & $95(17.7)$ & \\
\hline$\geq 72801$ & 6768 (2.5) & $6586 \quad(2.4)$ & $182(33.6)$ & & 1048 (32.6) & $872(32.5)$ & $176(32.8)$ & \\
\hline
\end{tabular}




\begin{tabular}{|c|c|c|c|c|c|c|c|}
\hline \multirow[b]{2}{*}{ Variable } & \multirow{2}{*}{$\begin{array}{c}\text { No. of } \\
\text { patients }\end{array}$} & \multicolumn{5}{|c|}{ Cancer stage, no. (\%) } & \multirow{2}{*}{$\begin{array}{c}p \\
\text { value }\end{array}$} \\
\hline & & 0 & 1 & II & III & IV & \\
\hline Total & 3216 & $280(8.7)$ & $831(25.8)$ & $660(20.5)$ & $778(24.2)$ & $667(20.7)$ & 0.48 \\
\hline Nonphysicians & 2680 & $233(8.7)$ & $692(25.8)$ & $543(20.3)$ & $664(24.8)$ & $548(20.4)$ & \\
\hline Physicians & 536 & $47 \quad(8.8)$ & $139(25.9)$ & $117(21.8)$ & $114(21.3)$ & $119(22.2)$ & \\
\hline \multicolumn{8}{|l|}{ Sex } \\
\hline Male & & & & & & & 0.4 \\
\hline Nonphysicians & 1975 & 71 (3.6) & $475(24.0)$ & $355(18.0)$ & $590(29.9)$ & $484(24.5)$ & \\
\hline Physicians & 394 & $12(3.0)$ & $102(25.9)$ & $77(19.5)$ & $100(25.4)$ & $103(26.1)$ & \\
\hline Female & & & & & & & 0.8 \\
\hline Nonphysicians & 705 & $162(23.0)$ & $217(30.8)$ & $188(26.7)$ & $74(10.5)$ & $64(9.1)$ & \\
\hline Physicians & 142 & $35(24.6)$ & $37(26.0)$ & $40(28.2)$ & $14(9.9)$ & $16(11.3)$ & \\
\hline \multicolumn{8}{|l|}{ Age, yr } \\
\hline $25-44$ & & & & & & & 0.4 \\
\hline Nonphysicians & 498 & 89 (17.9) & $144(28.9)$ & $104(20.9)$ & $94(18.9)$ & 67 (13.4) & \\
\hline Physicians & 99 & $24(24.2)$ & $26(26.3)$ & $23(23.2)$ & $12(12.1)$ & $14(14.1)$ & \\
\hline $45-54$ & & & & & & & 0.8 \\
\hline Nonphysicians & 642 & $83(12.9)$ & $178(27.7)$ & $139(21.6)$ & $127(19.8)$ & $115(17.9)$ & \\
\hline Physicians & 131 & $13(9.9)$ & $40(30.5)$ & $31(23.7)$ & $23(17.6)$ & $24(18.3)$ & \\
\hline $55-64$ & & & & & & & 0.8 \\
\hline Nonphysicians & 658 & $39(5.9)$ & $196(29.8)$ & $136(20.7)$ & $169(25.7)$ & $118(17.9)$ & \\
\hline Physicians & 133 & $6(4.5)$ & $34(25.6)$ & $31(23.3)$ & $35(26.3)$ & $27(20.3)$ & \\
\hline$\geq 65$ & & & & & & & 0.6 \\
\hline Nonphysicians & 882 & $22(2.5)$ & $174(19.7)$ & $164(18.6)$ & $274(31.1)$ & $248(28.1)$ & \\
\hline Physicians & 173 & $4(2.3)$ & $39(22.5)$ & $32(18.5)$ & $44(25.4)$ & $54(31.2)$ & \\
\hline \multicolumn{8}{|c|}{ Residence, level of urbanization } \\
\hline Level 1 & & & & & & & 0.8 \\
\hline Nonphysicians & 1000 & $95(9.5)$ & $264(26.4)$ & $214(21.4)$ & $249(24.9)$ & $178(17.8)$ & \\
\hline Physicians & 199 & $18(9.0)$ & $50(25.1)$ & $48(24.1)$ & $44(22.1)$ & $39(19.6)$ & \\
\hline Levels 2 and 3 & & & & & & & 0.9 \\
\hline Nonphysicians & 1161 & $104(9.0)$ & $289(24.9)$ & $237(20.4)$ & $280(24.1)$ & $251(21.6)$ & \\
\hline Physicians & 232 & $21(9.0)$ & $59(25.4)$ & $50(21.5)$ & $50(21.5)$ & $52(22.4)$ & \\
\hline Levels 4 and 5 & & & & & & & 0.3 \\
\hline Nonphysicians & 366 & $22(6.0)$ & $101(27.6)$ & $65(17.8)$ & $97(26.5)$ & $81(22.1)$ & \\
\hline Physicians & 74 & $3(4.0)$ & $24(32.4)$ & $9(12.2)$ & $15(20.3)$ & $23(31.1)$ & \\
\hline Levels 6 and 7 & & & & & & & 0.2 \\
\hline Nonphysicians & 153 & $12(7.8)$ & $38(24.8)$ & 27 (17.6) & $38(24.8)$ & $38(24.8)$ & \\
\hline Physicians & 31 & $5(16.1)$ & $6(19.3)$ & $10(32.3)$ & $5(16.1)$ & $5(16.1)$ & \\
\hline Monthly income, NT\$ & & & & & & & \\
\hline$\leq 17280^{\circ}$ & & & & & & & 0.98 \\
\hline Nonphysicians & 130 & $8(6.1)$ & $36(27.7)$ & $31(23.8)$ & $30(23.1)$ & $25(19.2)$ & \\
\hline Physicians & 26 & $2(7.7)$ & $8(30.8)$ & $5(19.2)$ & $6(23.1)$ & $5(19.2)$ & \\
\hline $17281-36300$ & & & & & & & 0.7 \\
\hline Nonphysicians & 525 & $48(9.1)$ & $101(19.2)$ & $110(20.9)$ & $137(26.1)$ & $129(24.6)$ & \\
\hline Physicians & 105 & $10(9.5)$ & $23(21.9)$ & $24(22.9)$ & $20(19.0)$ & $28(26.7)$ & \\
\hline $36301-45800$ & & & & & & & 0.6 \\
\hline Nonphysicians & 474 & $38(8.0)$ & $107(22.6)$ & $86(18.1)$ & $136(28.7)$ & $107(22.6)$ & \\
\hline Physicians & 94 & 7 (7.4) & $19(20.2)$ & $24(25.5)$ & $25(26.6)$ & $19(20.2)$ & \\
\hline $45801-57800$ & & & & & & & 0.5 \\
\hline Nonphysicians & 199 & $19(9.5)$ & $51(25.6)$ & 37 (18.6) & $50(25.1)$ & $42(21.1)$ & \\
\hline Physicians & 40 & $4(10.0)$ & $15(37.5)$ & $8(20.0)$ & $7(17.5)$ & $6(15.0)$ & \\
\hline $57801-72800$ & & & & & & & 0.3 \\
\hline Nonphysicians & 480 & $35(7.3)$ & $130(27.1)$ & $100(20.8)$ & $114(23.7)$ & $101(21.0)$ & \\
\hline Physicians & 95 & 7 (7.4) & $21(22.1)$ & $14(14.7)$ & $25(26.3)$ & $28(29.5)$ & \\
\hline 72801 & & & & & & & 0.6 \\
\hline Nonphysicians & 872 & $85(9.7)$ & $267(30.6)$ & $179(20.5)$ & $197(22.6)$ & $144(16.5)$ & \\
\hline Physicians & 176 & $17(9.7)$ & $53(30.1)$ & $42(23.9)$ & $31(17.6)$ & $33(18.7)$ & \\
\hline No catastrophic illness & & & & & & & 0.5 \\
\hline Nonphysicians & 2586 & $230(8.9)$ & $664(25.7)$ & $524(20.3)$ & $647(25.0)$ & $521(20.1)$ & \\
\hline Physicians & 517 & $44 \quad(8.5)$ & $136(26.3)$ & $112(21.7)$ & $111(21.5)$ & $114(22.0)$ & \\
\hline Catastrophic illness & & & & & & & 0.2 \\
\hline Nonphysicians & 94 & $3 \quad(3.2)$ & $28(29.8)$ & $19(20.2)$ & $17(18.1)$ & $27(28.7)$ & \\
\hline Physicians & 19 & $3(15.8)$ & $3(15.8)$ & $5(26.3)$ & $3(15.8)$ & $5(26.3)$ & \\
\hline CCI score & & & & & & & \\
\hline 0 & & & & & & & 0.2 \\
\hline Nonphysicians & 801 & $138(17.2)$ & $218(27.2)$ & $197(24.6)$ & $176(22.0)$ & $72(9.0)$ & \\
\hline Physicians & 176 & $29(16.5)$ & $49(27.8)$ & $43(24.4)$ & $30(17.0)$ & $25(14.2)$ & \\
\hline $1-3$ & & & & & & & 0.6 \\
\hline Nonphysicians & 1130 & $83(7.3)$ & $340(30.1)$ & $242(21.4)$ & $306(27.1)$ & $159(14.1)$ & \\
\hline Physicians & 216 & $16(7.4)$ & $74(34.3)$ & $49(22.7)$ & $54(25.0)$ & $23(10.6)$ & \\
\hline $4-6$ & & & & & & & 0.05 \\
\hline Nonphysicians & 389 & $11(2.8)$ & $94(24.2)$ & $63(16.2)$ & $113(29.1)$ & $108(27.8)$ & \\
\hline Physicians & 67 & 1 (1.5) & $10(14.9)$ & $19(28.4)$ & $14(20.9)$ & $23(34.3)$ & \\
\hline$\geq 7$ & & & & & & & 0.5 \\
\hline Nonphysicians & 360 & $1 \quad(0.3)$ & $40(11.1)$ & $41(11.4)$ & 69 (19.2) & $209(58.1)$ & \\
\hline Physicians & 77 & $1(1.3)$ & $6(7.8)$ & $6 \quad(7.8)$ & $16(20.8)$ & $48(62.3)$ & \\
\hline
\end{tabular}


compared with their nonphysician counterparts matched for age, sex, residence and income. The findings were consistent with extensive subgroup comparisons for cancer stage distribution (Table 2) and under multinomial logistic regression and sensitivity analyses (Table 4).

Being a physician confers several factors that influence health: high socioeconomic status, increased knowledge about health care, heavy workload and a high-stress working environment. ${ }^{5,-11,26-29}$ After propensity score matching, our physician cohort and nonphysician control group were socioeconomically similar. Thus, the two groups may have differed in terms of knowledge, attitude and practice of health care, or workload. The failure to diagnose cancer at earlier stages in physicians suggests that they may have gaps in practising a healthy lifestyle, which deserve further attention.

Female physicians in our cohort had higher risks for advanced cancer stages at diagnosis for breast and cervical cancers. Sankila and colleagues have previously reported that the cancer risk of female health care personnel was increased when compared with that of all economically active women ${ }^{30}$ and Chiang and colleagues have reported that only $12 \%$ of female medical personnel under- went mammography and only $10 \%$ underwent clinical examinations as recommended. ${ }^{31}$ These studies suggest that certain obstacles may exist for female physicians to undergo screening, which could explain why female physicians have a higher risk of advanced cancer at diagnosis.

Attending screening services is important in the early detection of cancer. Women who consistently underwent mammography screening as recommended were more likely to receive a diagnosis during the earlier stages of breast cancer. ${ }^{13}$ In Taiwan, the percentage of advanced stages of cervical cancer have declined since the implementation of a national screening program in $1995 .{ }^{12,14}$ However, physicians appear to have mediocre participation in screening. Gross and colleagues reported that $35 \%$ of physicians they followed in the United States did not have a regular source of health care, suggesting that the physicians were not undergoing recommended screening for breast, colon or prostate cancer. ${ }^{32}$ In Israel, $59.4 \%$ of family physicians reported a strong belief in the importance of screening tests, but only $27.5 \%$ underwent such tests themselves. ${ }^{33}$ In Canada, although $48 \%$ of physicians have undergone a clinical breast or testicular examination, $39 \%$ of doctors had not received

Table 3: Relative risk of differences in cancer stages at diagnosis

\begin{tabular}{|c|c|c|c|c|c|c|}
\hline \multirow[b]{2}{*}{ Comparison } & \multicolumn{3}{|c|}{ All physicians } & \multicolumn{3}{|c|}{ Western physicians } \\
\hline & \multicolumn{2}{|c|}{ OR* $(95 \% \mathrm{Cl})$} & $p$ value & \multicolumn{2}{|c|}{$O R *(95 \% \mathrm{Cl})$} & $p$ value \\
\hline \multicolumn{7}{|l|}{ All included cancers } \\
\hline Stage $0 \mathrm{v}$. $\geq$ stage I & 0.99 & $(0.71-1.38)$ & 0.95 & 1.00 & $(0.69-1.45)$ & 0.99 \\
\hline$\leq$ stage I v. $\geq$ stage II & 0.99 & $(0.82-1.21)$ & 0.9 & 0.93 & $(0.75-1.16)$ & 0.5 \\
\hline$\leq$ stage II v. $\geq$ stage III & 0.93 & $(0.77-1.12)$ & 0.5 & 0.95 & $(0.77-1.18)$ & 0.7 \\
\hline$\leq$ stage III v. stage IV & 1.11 & $(0.89-1.39)$ & 0.4 & 1.20 & $(0.94-1.54)$ & 0.1 \\
\hline \multicolumn{7}{|c|}{ Included cancerst excluding female-only cancersł } \\
\hline Stage $0 \mathrm{v}$. $\geq$ stage I & 1.01 & $(0.58-1.78)$ & 0.96 & 1.19 & $(0.61-2.32)$ & 0.6 \\
\hline$\leq$ stage I v. $\geq$ stage II & 0.93 & $(0.74-1.17)$ & 0.5 & 0.86 & $(0.67-1.11)$ & 0.2 \\
\hline$\leq$ stage II v. $\geq$ stage III & 0.89 & $(0.73-1.10)$ & 0.3 & 0.87 & $(0.69-1.10)$ & 0.2 \\
\hline s stage III v. stage IV & 1.04 & $(0.82-1.33)$ & 0.7 & 1.10 & $(0.84-1.43)$ & 0.5 \\
\hline \multicolumn{7}{|l|}{ Female-only cancers: } \\
\hline Stage $0 \mathrm{v}$. $\geq$ stage I & 0.97 & $(0.62-1.52)$ & 0.9 & 0.75 & $(0.45-1.25)$ & 0.3 \\
\hline$\leq$ stage I v. $\geq$ stage II & 1.21 & $(0.81-1.82)$ & 0.3 & 1.02 & $(0.62-1.68)$ & 0.9 \\
\hline$\leq$ stage II v. $\geq$ stage III & 1.24 & $(0.66-2.30)$ & 0.5 & 1.22 & $(0.58-2.58)$ & 0.6 \\
\hline$\leq$ stage III v. stage IV & 2.64 & $(1.20-5.81)$ & 0.02 & 2.87 & $(1.17-7.05)$ & 0.02 \\
\hline \multicolumn{7}{|c|}{$\begin{array}{l}\text { Note: } \mathrm{Cl}=\text { confidence interval, } \mathrm{OR}=\text { odds ratio. } \\
\text { *Reference group: nonphysicians. } \\
\text { tLung cancer, colorectal cancer, oral cancer and hepatoma. } \\
\text { †Female breast and cervical cancer. }\end{array}$} \\
\hline
\end{tabular}


Table 4: Relative adjusted* risk of differences in cancer stage at diagnosis

\begin{tabular}{|c|c|c|c|c|c|c|}
\hline \multirow[b]{2}{*}{ Comparison } & \multicolumn{3}{|c|}{ All physicians } & \multicolumn{3}{|c|}{ Western physicians } \\
\hline & \multicolumn{2}{|c|}{ Adjusted OR† $(95 \% \mathrm{Cl})$} & $p$ value & \multicolumn{2}{|c|}{ Adjusted OR† (95\% Cl) } & \multirow[t]{2}{*}{$p$ value } \\
\hline \multicolumn{6}{|l|}{ All included cancers } & \\
\hline Stage $0 \mathrm{v} . \geq$ stage I & 0.99 & $(0.70-1.41)$ & 0.96 & 0.91 & $(0.61-1.35)$ & 0.6 \\
\hline$\leq$ stage I v. $\geq$ stage II & 0.99 & $(0.81-1.22)$ & 0.9 & 0.88 & $(0.70-1.11)$ & 0.3 \\
\hline$\leq$ stage II v. $\geq$ stage III & 0.92 & $(0.75-1.13)$ & 0.4 & 0.88 & $(0.70-1.11)$ & 0.3 \\
\hline$\leq$ stage III v. stage IV & 1.14 & $(0.89-1.46)$ & 0.3 & 1.18 & $(0.90-1.55)$ & 0.2 \\
\hline \multicolumn{7}{|c|}{ Included cancers $¥$ excluding female-only cancers } \\
\hline Stage $0 \mathrm{v}$. $\geq$ stage I & 1.03 & $(0.58-1.82)$ & 0.9 & 1.17 & $(0.60-2.31)$ & 0.6 \\
\hline$\leq$ stage I v. $\geq$ stage II & 0.93 & $(0.73-1.17)$ & 0.5 & 0.83 & $(0.64-1.08)$ & 0.2 \\
\hline$\leq$ stage II v. $\geq$ stage III & 0.89 & $(0.71-1.10)$ & 0.3 & 0.84 & $(0.66-1.07)$ & 0.2 \\
\hline$\leq$ stage III v. stage IV & 1.06 & $(0.82-1.38)$ & 0.7 & 1.09 & $(0.82-1.45)$ & 0.6 \\
\hline \multicolumn{7}{|l|}{ Female-only cancers§ } \\
\hline Stage $0 \mathrm{v} . \geq$ stage I & 0.91 & $(0.57-1.46)$ & 0.7 & 0.69 & $(0.41-1.19)$ & 0.2 \\
\hline$\leq$ stage I v. $\geq$ stage II & 1.12 & $(0.73-1.71)$ & 0.6 & 0.91 & $(0.54-1.53)$ & 0.7 \\
\hline$\leq$ stage II v. $\geq$ stage III & 1.09 & $(0.57-2.11)$ & 0.8 & 1.02 & $(0.46-2.26)$ & 0.96 \\
\hline$\leq$ stage III v. stage IV & 2.37 & $(1.01-5.57)$ & 0.048 & 2.46 & $(0.92-6.58)$ & 0.07 \\
\hline \multicolumn{7}{|c|}{$\begin{array}{l}\text { Note: } \mathrm{Cl}=\text { confidence interval, } \mathrm{OR}=\text { odds ratio. } \\
\text { *Models were controlled for age, sex (with the exception of the female-only cancers), income, residence, catastrophic illness } \\
\text { and Charlson comorbidity index. } \\
\text { tReference group: nonphysicians. } \\
\text { f Lung cancer, colorectal cancer, oral cancer and hepatoma. } \\
\text { SFemale breast cancer and cervical cancer. }\end{array}$} \\
\hline
\end{tabular}

such an examination for more than 5 years or had never received one. ${ }^{34}$ Further research may be needed to investigate physician participation in screening.

Access to a primary care physician may be an additional factor related to cancer being diagnosed at an earlier stage. An adequate supply of primary care physicians had protective effects on breast cancer stage and survival in Ontario, ${ }^{15}$ and higher physician density was associated with lower incidence of late-stage colorectal cancer in Pennsylvania. ${ }^{16}$ Furthermore, physicians, with their medical networks and better access to proper care, have been shown to have better health outcomes then nonphysicians. Shen and colleagues reported that physicians are less likely to have or die of severe sepsis with acute severe infections, and better medical knowledge, higher disease awareness and easier access to health care were considered as related factors. ${ }^{10}$ In addition, Ridker and colleagues attributed a lower risk of death from acute myocardial infarction in male physicians to the shorter time delay from disease onset to hospital admission. ${ }^{11}$

Physicians in both Canada and Taiwan have reported their workload as too heavy; ${ }^{5,26}$ however, the workloads of physicians under different health care systems differ substantially. This is a potential avenue for further investigation.

\section{Limitations}

Neither database provided important information on cancer-related personal health behaviours. However, the propensity score adjustment is an important statistical technique to reduce the bias from confounding variables in observational studies and mimic the results of randomized control tests. ${ }^{35}$ We used the propensity score method with extensive matches including income and environmental factors to reduce potential confounding factors. In addition, the long follow-up and nationwide design provided adequate power.

Thyroid cancer and prostate cancer are both reported to be common cancers in physicians; ${ }^{1}$ however, because their rankings in the general population are not high enough to be included in the long form of the Taiwan Cancer Registry File, these cancers were not included in our analysis.

\section{Conclusion}

Cancer is not diagnosed at earlier stages in physicians than in nonphysicians in Taiwan. However, female physicians are more likely to have 
more advanced stages of breast and cervical cancer at diagnosis.

\section{References}

1. Lin SY, Lin CL, Hsu WH, et al. A comparison of cancer incidence among physician specialists and the general population: a Taiwanese cohort study. J Occup Health 2013;55:158-66.

2. Eriksson M, Hardell L, Malker $\mathrm{H}$, et al. Increased cancer incidence in physicians, dentists, and health care workers. Oncol Rep 1998;5: 1413-8.

3. Maître A, Colonna M, Gressin C, et al. Increased incidence of haematological cancer among physicians in a University Hospital. Int Arch Occup Environ Health 2003;76:24-8.

4. Nurminen M, Rimpela A, Pukkala E. Exceptionally low cancer incidence in doctors. Lancet 1988;1:190-1.

5. CMA Guide to physician health and well-being: facts, advice and resources for Canadian doctors. Ottawa: Canadian Medica Association; 2003.

6. Arnetz BB. Psychosocial challenges facing physicians of today. Soc Sci Med 2001;52:203-13.

7. Pullen D, Lonie CE, Lyle DM, et al. Medical care of doctors. Med J Aust 1995;162:481-4.

8. Uallachain GN. Attitudes towards self-health care: a survey of GP trainees. Ir Med J 2007;100:489-91.

9. Innos K, Rahu K, Baburin A, et al. Cancer incidence and causespecific mortality in male and female physicians: a cohort study in Estonia. Scand J Public Health 2002;30:133-40.

10. Shen HN, Lu CL, Li CY. Do physicians have lower risk of severe sepsis and associated mortality? A matched cohort study. Crit Care Med 2014;42:816-23.

11. Ridker PM, Manson JE, Goldhaber SZ, et al. Comparison of delay times to hospital presentation for physicians and nonphysicians with acute myocardial infarction. Am J Cardiol 1992;70:10-3.

12. Chiang CJ, Chen YC, Chen CJ, et al. Cancer trends in Taiwan. Jpn J Clin Oncol 2010;40:897-904.

13. Vyas A, Madhavan S, Sambamoorthi U. Association between persistence with mammography screening and stage at diagnosis among elderly women diagnosed with breast cancer. Breast Cancer Res Treat 2014;148:645-54.

14. Chen YY, You SL, Chen CA, et al. Effectiveness of national cervical cancer screening programme in Taiwan: 12-year experiences. Br J Cancer 2009;101:174-7.

15. Gorey KM, Luginaah IN, Holowaty EJ, et al. Associations of physician supplies with breast cancer stage at diagnosis and survival in Ontario, 1988 to 2006. Cancer 2009;115:3563-70.

16. Ananthakrishnan AN, Hoffmann RG, Saeian K. Higher physician density is associated with lower incidence of late-stage colorectal cancer. J Gen Intern Med 2010;25:1164-71.

17. 2014-2015 National Health Insurance Annual Report. Taipei: National Health Insurance Administration, Ministry of Health and Welfare, Executive Yuan; 2014: 51. Available: www.nhi. gov.tw/Resource/webdata/13767_1_NHI2014-2015\%20Annual\% 20Report\%20(bilingual)_141230.pdf (accessed 2015 Apr. 29).

18. Taiwan Cancer registry introduction. Taipei: Taiwan Health Promotion Administration; 2012. Available: http://tcr.cph.ntu. edu.tw/main.php?Page=N1 (accessed 2015 Mar. 15).

19. Taiwan Bureau of Health Promotion. [Taiwan cancer registry database]. Taipei: Taiwan Bureau of Health Promotion; 2010. Available: http://tcr.cph.ntu.edu.tw/uploadimages/CA10_LF97 .pdf (accessed 2015 Mar. 15)

20. Taiwan Cancer Registry coding manual long form revision 2011v.4. Taiwan Health Promotion Administration; 2015:16-35, 113. Available: http://tcr.cph.ntu.edu.tw/uploadimages/Longform \%20Manual_Official\%20version_20150105_W.pdf (accessed 2015 Mar. 15).

21. Cancer staging references. Chicago: American Joint Commitee on Cancer; 2015. Available: https://cancerstaging.org/ references-tools/Pages/What-is-Cancer-Staging.aspx (accessed 2015 June 10).

22. Liu CY, Hung YT, Chuang YL, et al. Incorporating development stratification of Taiwan Townships into sampling design of large scale health interview survey. J Health Manag 2006;4:1-22.
23. Deyo RA, Cherkin DC, Ciol MA. Adapting a clinical comorbidity index for use with ICD-9-CM administrative databases. $J$ Clin Epidemiol 1992;45:613-9.

24. Regulations governing the exemption of the national health insurance beneficiaries from the co-payment. Taipei: Taiwan National Health Insurance Administration; 2011. Available: www.nhi.gov. tw/English/webdata/webdata.aspx?menu=11\&menu id=295\&WD ID=295\&webdata id=2431 (accessed 2015 Mar. 15).

25. Kleinbaum DG, Klein M. Logistic regression: a self-learning text. 3rd ed. New York: Springer-Verlag; 2010.

26. Lu JF, Hsiao WC. Does universal health insurance make health care unaffordable? Lessons from Taiwan. Health Aff (Millwood) 2003;22:77-88

27. Spurgeon P, Barwell F, Maxwell R. Types of work stress and implications for the role of general practitioners. Health Serv Manage Res 1995;8:186-97.

28. Sutherland VJ, Cooper CL. Identifying distress among general practitioners: predictors of psychological ill-health and job dissatisfaction. Soc Sci Med 1993;37:575-81.

29. Landrigan CP, Rothschild JM, Cronin JW, et al. Effect of reducing interns' work hours on serious medical errors in intensive care units. N Engl J Med 2004;351:1838-48.

30. Sankila R, Karjalainen S, Laara E, et al. Cancer risk among health care personnel in Finland, 1971-1980. Scand J Work Environ Health 1990;16:252-7.

31. Chiang TT, Tai CS, Lin PH. To survey the implementation of breast cancer screening hehaviors of female medical personnel - based on health belief model. VGH Nursing 2011;28:130-8.

32. Gross CP, Mead LA, Ford DE, et al. Physician, heal thyself? Regular source of care and use of preventive health services among physicians. Arch Intern Med 2000;160:3209-14.

33. Peleg R, Ostermich A, Gienco V, et al. Screening tests among family doctors: do we do as we preach? Public Health 2013 127:282-9.

34. Frank E, Segura C. Health practices of Canadian physicians. Can Fam Physician 2009;55:810-11.e7.

35. Austin PC. An introduction to propensity score methods for reducing the effects of confounding in observational studies. Multivariate Behav Res 2011;46:399-424.

Affiliations: Department of Public Health and Department of Health Services Administration (Hsu), China Medical University, Taichung, Taiwan; Department of Internal Medicine (Hsu), Ditmanson Medical Foundation Chia-Yi Christian Hospital, Chia-Yi, Taiwan; Department of Healthcare Administration (Kung), Asia University, Taichung, Taiwan; School of Health Care Administration, Health Policy and Care Research Center (Chang), Taipei Medical University, Taipei, Taiwan; Department of Health Services Administration (Wang, Tsai), China Medical University, Taichung, Taiwan.

Contributors: Yueh-Han Hsu, Pei-Tseng Kung and WenChen Tsai conceived and designed the study. Yueh-Hsin Wang and Wen-Chen Tsai collected the data. Yueh-Han Hsu, Pei-Tseng Kung, Yao-Mao Chang and Wen-Chen Tsai analyzed and interpreted the data. All of the authors drafted the manuscript and revised it for important intellectual content. All of the authors approved the final version for publication and agree to act as guarantors of the work.

Funding: This study was supported by grants (CYCH R10130, CMU101-ASIA-14) from Ditmanson Medical Foundation Chia-Yi Christian Hospital, China Medical University, and Asia University.

Data sharing: Data are available upon written request to the corresponding author.

Acknowledgements: The authors thank the Statistics Center of the Ministry of Health and Welfare for use of the National Health Insurance Research Database and the Taiwan Cancer Registry File. 